\title{
Pancreatic cancer treatment after FOLFIRINOX: prognostic importance of chemotherapy dose intensity and albumin/ globulin ratio in second line
}

\author{
(DÖzgen Ahmet Yıldırım', DErkan Erdur² \\ ${ }^{1}$ University of Health Sciences, Dr. Abdurrahman Yurtarslan Ankara Oncology Training and Research Hospital, Deparment of Medical Oncology, Ankara, Turkey \\ ${ }^{2}$ University of Health Sciences, Gazi Yaşargil Training and Research Hospital, Department of Internal Medicine, Division of Medical Oncology, Diyarbakır, Turkey
}

Cite this article as: Yıldırım ÖA, Erdur E. Pancreatic cancer treatment after FOLFIRINOX: prognostic importance of chemotherapy dose intensity and albumin/globulin ratio in second line. J Health Sci Med 2022; 5(1): 156-160.

\begin{abstract}
Aim: Pancreatic adenocarcinoma (PA) is the seventh most common cause of cancer-related mortality. Our primary endpoint of study was to determine the relationship between albumin/globulin ratio (AGR) and progression-free and overall survival (PFS and OS) in second-line treatment after FOLFIRINOX. Our secondary endpoint was to assess treatment side effects and the relationship of treatment dose intensity with treatment type and AGR.

Material and Method: PA patients who followed-up between January 2014 and January 2021 were evaluated retrospectively. Age, gender, ECOG score and AGR recorded at the beginning of the second-line treatment. Thrombocytopenia, neutropenia, chemotherapy type, chemotherapy dose intensity, PFS and OS were recorded during the second-line treatment.

Results: Median age 64 (44-80), 72 (70.6\%) male, 102 metastatic PA patients were evaluated. 76 (74.5\%) patients were ECOG 0-1, $26(25.5 \%)$ patients were ECOG 2. Of these patients in the second step, 68 (66.7\%) received single-agent gemcitabine and $34(33.3 \%)$ received Nab-paclitaxel + gemcitabine treatment. Progression and exitus events occurred in all cases. Median PFS was 166.8 days in the AGR $>1.2$ group, it was 80.7 days in the AGR $<1.2$ group ( $\mathrm{p}=0.003)$. While the median OS was 295.7 days in the AGR $>1.2$ group, it was 144 days in the $A G R<1.2$ group ( $p=0.041$ ). Dose intensity was $80.7 \%$ in the $A G R>1.2$ group, it was $71.3 \%$ in the $A G R<1.2$ group ( $\mathrm{p}=0.002$ ).

Conclusion: Even with palliative and advanced treatment, achieving a dose density close to $80 \%$ is a good prognostic indicator. AGR is a prognostic marker that remains effective in advanced stages of PA.
\end{abstract}

Keywords: Pancreatic cancer, dose intensity, albumin globulin ratio

\section{INTRODUCTION}

Pancreatic adenocarcinoma (PA) is the seventh most common cause of cancer-related mortality. It is observed in Europe, North America and Oceania, with the highest incidence at 7-10/10000 levels. Globocan data predicts that the incidence will double in 2040 (1). Incidence and mortality increases with age and peak just above 70 years of age. PA has poor prognosis, due to its asymptomatic early-stage clinical course and the weak systemic treatment efficacy compared to many other cancer types (2). Smoking, diabetes, obesity, advanced age, ethnic and genetic factors, Helicobacter pylori infection, non-0 blood type and chronic pancreatitis are the main risk factors. PA is mostly associated with environmental factors. PA constitutes $95 \%$ of pancreatic cancers (3). PA is a difficult cancer to diagnose at an early stage. Jaundice, pain, acholic stools, and signs of fatigue often accompany advanced disease. $85 \%$ of the cases are diagnosed in the locally advanced or metastatic stage and shows a very low 5-year survival rate (4). The rate of familial and genetically transmitted diseases is low. Among germline mutations, ATM, BRCA1, BRCA2, CDKN2A, PALB2, PRSS1, STK11, TP53, and Lynch syndrome related mismatch repair defects have been shown (5).

The treatment approach should be multimodal. FOLFIRINOX is the most effective treatment regimen based on available data, following surgery in resectable disease. In totally resected cases, a 3-year survival of over $60 \%$ can be achieved with adjuvant FOLFIRINOX regimen. However, FOLFIRINOX is not a regimen that 
can be tolerated by all patients. The therapeutic benefit of single agent gemcitabine therapy is well established. It has been found that neoadjuvant FOLFIRINOX treatment increases the chance of resectability in borderline resectable cases (6-8).

Progression-free survival (PFS) and overall survival (OS) superiority of FOLFIRINOX to single-agent Gemcitabine has also been demonstrated in metastatic disease. Similarly, the combination of Nab-paclitaxel with gemcitabine has shown superior results to the single agent gemcitabine. Toxicity was observed more frequently in combination regimens and more frequent dose reductions were required $(9,10)$.

Contrary to first-line therapy, comparative studies of second-line therapeutic approaches in PA are limited. Almost all patients who receive potent and toxic combined regimens such as FOLFIRINOX in the first line, receive single-agent therapy in the second line. At this stage, a limited number of patients can tolerate the combination of Nab-paclitaxel + gemcitabine (11). OS benefit was demonstrated in the NAPOLI-1 study, in which the combination of nanoliposomal irinotecan and fluorouracil was evaluated as a second step in cases who received a gemcitabine-based combined regimen in the first step. The association of this benefit with the general quality of life benefit has also been demonstrated $(12,13)$.

Dose density is a parameter with well-proven prognostic value. In a study evaluating the relationship between dose intensity of FOLFIRINOX treatment and survival in pancreatic and other gastrointestinal cancers; relative dose intensity cut-off of $77-79 \%$ was found to be significant in terms of PFS and OS results (14).

Albumin and globulin are the main protein components of human serum. Albumin has been shown to be a prognostic marker in many cancer types, both showing the nutritional status and being a negative acute phase reactant (15). Globulin plays a critical role in immunity and inflammation with its cortisol-binding function (16). The relationship between albumin globulin ratio (AGR) and cancer prognosis are still a controversial issue in the literature. A meta-analysis published in 2018 found an association between low pretreatment AGR and low OS and PFS in many cancer types. (17).

In order to show that AGR is a practical and an inexpensive tool in terms of predictive prognostic value and treatment planning, we aimed to evaluate its relationship with chemotherapy dose intensity, which is a well-proven prognostic predictor.

In our study, we aimed to evaluate the second-line cases after FOLFIRINOX in metastatic pancreatic cancer, to discuss clinical and laboratory data and treatment results, retrospectively. At this point, our primary endpoint is to determine the relationship of patients' AGR with PFS and OS. Our secondary endpoint is to assess treatment side effects and the relationship of treatment dose intensity with treatment type and AGR.

\section{MATERIAL AND METHOD}

Within the scope of ethics committee approval for retrospective studies, approval was obtained from the Ethics Committee of Health Sciences University Gazi Yaşargil Training and Research Hospital (Date: 08.10.2021, Decision No: 896). All procedures were carried out in accordance with the ethical rules and the principles of the Declaration of Helsinki.

Patients with a diagnosis of PA followed-up in the Medical Oncology Department of Gazi Yaşargil Training and Research Hospital between January 2014 and January 2021 were evaluated retrospectively. Inclusion criterias; being between the ages of 18 and 70 , being inoperable at diagnosis and not suitable for local treatments, having completed FOLFIRINOX chemotherapy in the first line therapy, relapsed within the first 1 year after the end of the 12 cycles of FOLFIRINOX treatment and applied second-line treatment, and being able to receive second-line treatment for at least 3 months.

For the FOLFIRINOX regimen, standard oxaliplatin 85 $\mathrm{mg} / \mathrm{m} 2$, irinotecan $180 \mathrm{mg} / \mathrm{m} 2$, leucovorin $400 \mathrm{mg} / \mathrm{m} 2$, and fluorouracil $400 \mathrm{mg} / \mathrm{m} 2$ IV push followed by 2400 $\mathrm{mg} / \mathrm{m} 2$ 46-hour continuous IV infusion chemotherapy every 14 days was considered. The standard 1000 $\mathrm{mg} / \mathrm{m} 2$ 1-8-15/28-day regimen was accepted for the gemcitabine regimen. The nab-paclitaxel regimen was accepted as $125 \mathrm{mg} / \mathrm{m} 2$ in combination with the gemcitabine standard regimen for 1-8-15/28 days.

The parameters of the patients were evaluated retrospectively; including age, gender, initial ECOG score, initial AGR, thrombocytopenia, neutropenia, chemotherapy type, chemotherapy dose intensity (\%),PFS (days), and OS (days).

Patients were divided into two strata for AGR, $\geq 1.2$ and $<1.2$. The parameters of these two layers were analyzed comparatively. AGR was calculated by; serum albumin/ (serum total protein - serum albumin).

Chemotherapy dose intensity calculation was accepted as $100 \%$ in patients who were able to receive full dose in all sessions of chemotherapy administered in the first 3 months of the second line chemotherapy. In those who received missing session treatment due to delayed treatments in the first 3 months, the dose of that missing session was included in the dose intensity calculation 
as $0 \%$. Treatments after 3 months were not included in the dose intensity calculation, as it was concluded that the dose intensity-prognosis relationship could not be evaluated properly due to variable delays in the treatments after the first 3 months.

Patients with total bilirubin $>3$, presence of biliary stent, $>3$ ECOG score, creatinine levels above 1.4, presence of clinical conditions or complications that would interfere with oral feeding, presence of brain metastasis, and uncontrollable pain were excluded.

SPSS 26.0 (IBM Corporation, Armonk, New York, United States) program was used in the analysis of the variables. The conformity of univariate data to normal distribution was evaluated with the Shapiro-Wilk francia test, while homogeneity of variance was evaluated with the Levene test. In the comparison of two independent groups according to quantitative data, the IndependentSamples $\mathrm{T}$ test was used together with the Bootstrap results, while the Mann-Whitney $U$ test was used together with the Monte Carlo results. In the comparison of categorical variables with each other, Fisher Exact test exact results and Fisher-Freeman-Holton test was tested with Monte Carlo Simulation technique. Quantitative variables are mean \pm SD in tables. (standard deviation) and Median (Percentile 25\%/Percentile 75\%), while categorical variables were shown as $\mathrm{n}(\%)$. Variables were analyzed at $95 \%$ confidence level, and $\mathrm{p}$ value less than 0.05 was considered significant.

\section{RESULTS}

Our study included 102 metastatic PA patients with a median age of 64 (44-80), 72 (70.6\%) men, who met the criteria. All patients progressed between 3-12 months after completion of 12 cycles of FOLFIRINOX chemotherapy. 76 (74.5\%) patients were ECOG 0-1, $26(25.5 \%)$ patients were ECOG 2. Of these patients in the second line, $68(66.7 \%)$ received single-agent gemcitabine and $34(33.3 \%)$ received Nab-paclitaxel + gemcitabine (Table 1).

\begin{tabular}{|c|c|}
\hline Features & \\
\hline Age (median, min-max) & $64(44-80)$ \\
\hline Gender (female, $\mathrm{n}, \%$ ) & $30(29.4 \%)$ \\
\hline \multicolumn{2}{|l|}{ Ecog } \\
\hline $0-1(n, \%)$ & $76(74.5 \%)$ \\
\hline $2+(\mathrm{n}, \%)$ & $26(25.5 \%)$ \\
\hline \multicolumn{2}{|l|}{ Chemotherapy } \\
\hline Gemsitabin (n,\%) & $68(66.7 \%)$ \\
\hline Gemsitabin+nabpaklitaxel (n,\%) & $34(33.3 \%)$ \\
\hline
\end{tabular}

Comparative analysis of PFS and OS results in AGR $>1.2$ and $A G R<1.2$ groups of all cases with progression and exitus events was performed. Median PFS was 166.8 days in the AGR $>1.2$ group, it was 80.7 days in the AGR $<1.2$ group ( $\mathrm{p}=0.003)$. Median OS was 295.7 days in the AGR $>1.2$ group, it was 144 days in the AGR $<1.2$ group $(\mathrm{p}=0.041)$.

When we look at the chemotherapy dose density analysis with AGR; in both gemcitabine and gemcitabinenabpaclitaxel groups, it was observed that the AGR value above 1.2 was higher than the patients with AGR below $1.2(\mathrm{p}<0.001)$. Dose intensities in $\mathrm{AGR}>1.2$ and $<1.2$ groups were analyzed comparatively. While the dose intensity was $80.7 \%$ in the AGR $>1.2$ group, it was $71.3 \%$ in the $A G R<1.2$ group ( $\mathrm{p}=0.002)$. Dose intensity analysis was performed according to chemotherapy type. While the dose intensity was found to be 73.2 in the group receiving single-agent gemcitabine, it was found to be 79.6 in the nab-paclitaxel gemcitabine group and the difference was not statistically significant $(\mathrm{p}=0.07)$. In the gemcitabine group; dose intensity was 71.1 in the AGR $<1.2$ subgroup, and was 80.6 in the AGR $>1.2$ group $(\mathrm{p}=0.0019)$. In the gemcitabine nabpaklitaxel combination group; dose intensity was 73.5 in the AGR $<1.2$ subgroup, and was 80.8 in the AGR $>1.2$ group ( $\mathrm{p}=0.0028$ ) (Table 2 ). No statistically significant correlation was found between AGR and documented myelotoxidity (Table 3).

\begin{tabular}{|c|c|c|c|}
\hline & Gemsitabin & $\begin{array}{l}\text { Gemsitabin- } \\
\text { nabpaklitaxel }\end{array}$ & All patients \\
\hline AGR & $\begin{array}{c}\text { dose intensity } \\
\text { (mean/median } \\
\text { min-max) }\end{array}$ & $\begin{array}{c}\text { dose intensity } \\
\text { (mean/median min- } \\
\text { max) }\end{array}$ & $\begin{array}{c}\text { dose intensity } \\
\text { (mean/median min- } \\
\text { max) }\end{array}$ \\
\hline$<1.2$ & $\begin{array}{c}71.17 / 72 \\
(66-76)\end{array}$ & $\begin{array}{l}73.5 / 73 \\
(72-76)\end{array}$ & $\begin{array}{l}71.3 / 71 \\
(66-76)\end{array}$ \\
\hline$>1.2$ & $\begin{array}{c}80.60 / 80 \\
(74-90)\end{array}$ & $\begin{array}{l}80.8 / 81 \\
(76-88)\end{array}$ & $\begin{array}{l}80.7 / 80 \\
(74-90)\end{array}$ \\
\hline $\mathrm{p}$ value & 0.0019 & 0.0028 & 0.002 \\
\hline
\end{tabular}

Table 3. Relationship between albumin to globulin ratio (AGR), myelotoxicity

\begin{tabular}{|lllc|}
\hline Myelotoxicity & AGR $<\mathbf{1 . 2}$ & AGR $>\mathbf{1 . 2}$ & p value \\
\hline Trombocytopenia & & & 0.077 \\
Grad 1-2 (n, \%) & $35(61.4)$ & $35(77.8)$ & \\
Grad 3+ (n, \%) & $22(38.6)$ & $10(22.2)$ & \\
Neutropenia & & & 0.296 \\
Grad 1-3 (n, \%) & $27(47.4)$ & $26(57.8)$ & \\
Grad 4 (n, \%) & $30(52.6)$ & $19(42.2)$ & \\
\hline
\end{tabular}

\section{DISCUSSION}

In our knowledge, our study is the first in the literature that evaluate the relationship between AGR and chemotherapy dose intensity. The data we have obtained may shed light on the predetermination of treatment tolerability in advanced line chemotherapy patients. 
The first-line therapy in the treatment of metastatic PA is the primary therapy on which the clinician focuses his expectation. Generally, in the first-line treatment, it is fought within the framework of the longest possible PFS and quality of life goal. In cases that have received combination regimens such as FOLFIRINOX and progressed early, second-line treatment planning brings a pessimistic view for the clinician. In most cases, second-line treatment focuses on symptom palliation and promoting quality of life within the short pre-terminal PFS. In previous studies in this context, the superiority of gemcitabine nabpaclitaxel combination over single-agent treatments is clear in patients with good tolerance after the first step (18). It is clear that practical prognostic markers are needed to identify palliative targets in this poorly prognostic entity.

While evaluating the second-line therapeutic factors in our study, the data of the first 3 months of the treatment were discussed. Performing a retrospective study in advanced treatment is not healthy because of patientbased prognosis and poor prognosis of PA. Therefore, we evaluated prognostic factors, such as dose intensity and inflammatory parameters based on the first 3 months period.

Although chemotherapy dose intensity is an element that is tried not to be compromised in adjuvant treatments, the prognostic benefit of providing optimal dose density for metastatic cases is clear. However, optimal dose density may cause fatal complications in patients with low bone marrow reserve (19). At this point, the clinician should not forget that the main goal of advanced chemotherapy is palliation and quality of life support.

In pancreatic cancer, together with more studied inflammatory markers, such as neutrophil lymphocyte ratio (NLR) and platelet lymphocyte ratio (PLR); low AGR levels has been shown to be poor prognostic for OS (20). In a recent study evaluating inflammatory parameters in pancreatic cancer, NLR and PLR were evaluated together. Both parameters were found to be an independent prognostic for OS (21). Since exitus and progression events occurred in all of the cases in our study, we performed our statistical evaluation directly on absolute PFS and OS data and found that AGR 1.2 level had a prognostic value. Tezuka et al. (22) evaluated AGR after neoadjuvant chemotherapy in borderline resectable pancreatic cancer, low AGR rate was associated with low OS. In a study investigating neoadjuvant chemotherapy in upper tract urethelial carcinomas, it was found that pre-treatment AGR level had no effect on long-term outcomes (23). However, the AGR level evaluated in this study belongs to chemotherapy-naive patients. In our study, AGR level was recorded before the second line, after FOLFIRINOX therapy. Therefore, in our study, we could achieve information about the metabolic reserve status after treatment.
There are additional poor prognostic factors that may affect the albumin level in pancreatic cancer. Biliary stasis and inflammation, which occurs while bilirubin levels are high, especially in the presence of occlusive icterus, decrease the level of albumin, which is a negative acute phase reactant. However, in a study conducted by Feng et al. (24) in 2018, it was concluded that pre-treatment bilirubin and albumin levels alone are not prognostic in advanced pancreatic cancer. However, the AGR rate is a relatively specific marker in the inflammation-synthesis cascade. Liu et al. (25) determined that AGR measured before treatment is an independent prognostic factor in metastatic gastric cancer. We emphasize that the prognostic correlation of these types of markers may increase in metastatic and advanced stage PA where catabolic processes are dominant.

The main limitation of our study is its retrospective design. Performing studies evaluating therapeutic results with a prospective method will provide stronger results. Stronger reference results can be obtained with a multicenter, multi-geographic study with a higher number of patients. Additional stratification of PFS results of first-line therapy may be beneficial in terms of reaching more reliable results.

\section{CONCLUSION}

As a result, advanced line treatment for this entity with a poor prognosis generally does not follow a satisfactory course. Even in palliative and advanced treatment, achieving a dose intensity close to $80 \%$ is a good prognostic indicator. AGR is a prognostic marker that remains effective in advanced stages of PA.

\section{ETHICAL DECLARATIONS}

Ethics Committee Approval: The study was carried out with the permission of Ethics Committee of Health Sciences University Gazi Yaşargil Training and Research Hospital (Date: 08.10.2021, Decision No: 896).

Informed Consent: Because the study was designed retrospectively, no written informed consent form was obtained from patients.

Referee Evaluation Process: Externally peer-reviewed.

Conflict of Interest Statement: The authors have no conflicts of interest to declare.

Financial Disclosure: The authors declared that this study has received no financial support.

Author Contributions: All of the authors declare that they have all participated in the design, execution, and analysis of the paper, and that they have approved the final version. 


\section{REFERENCES}

1. Bray F, Ferlay J, Soerjomataram I, Siegel RL, Torre LA, Jemal A. Global cancer statistics 2018: GLOBOCAN estimates of incidence and mortality worldwide for 36 cancers in 185 countries. CA Cancer J Clin 2018; 68: 394-424.

2. Rawla P, Sunkara T, Gaduputi V. Epidemiology of pancreatic cancer: global trends, etiology and risk factors. World J Oncol 2019; 10: 10-27.

3. Capasso M, Franceschi M, Rodriguez-Castro KI, et al. Epidemiology and risk factors of pancreatic cancer. Acta Bio Medica Atenei Parm 2018; 89: 141-6.

4. McGuigan A, Kelly P, Turkington RC, Jones C, Coleman HG, McCain RS. Pancreaticcancer: a review of clinical diagnosis, epidemiology, treatment and outcomes. World J Gastroenterol 2018; 24: 4846-61.

5. Stjepanovic N, Moreira L, Carneiro F, et al. Hereditary gastrointestinal cancers: ESMO Clinical Practice Guidelines for diagnosis, treatment and follow-up $\dagger$. Ann Oncol 2019; 30: 1558-71.

6. ESMO Guidelines Committee. eUpdate - Cancer of the Pancreas Treatment Recommendations, https://www.esmo.org/guidelines/ gastrointestinal-cancers/pancreatic-cancer/eupdate-cancer-ofthe-pancreas-treatment-recommendations; 2019; 1

7. Ghaneh P, Palmer DH, Cicconi S, et al. ESPAC-5F: Four-arm, prospective, multicenter, international randomized phase IItrial of immediate surgery compared with neoadjuvant gemcitabine pluscapecitabine (GEMCAP) or FOLFIRINOX or chemoradiotherapy (CRT) in patients with borderline resectable pancreatic cancer. J Clin Oncol 2020; 38: 4505.

8. Katz MHG, Shi Q, Meyers JP, et al. Alliance A021501: Preoperative mFOLFIRINOX or mFOLFIRINOX plus hypofractionated radiation therapy (RT) for borderline resectable (BR) adenocarcinoma of the pancreas. J Clin Oncol 2021; 39: 377.

9. Conroy T, Desseigne F, Ychou M, et al. FOLFIRINOX versus gemcitabine for metastatic pancreatic cancer. N Engl J Med 2011; 364: 1817-25.

10. Von Hoff DD, Ervin T, Arena FP, et al. Increased survival in pancreatic cancer with nab-paclitaxel plus gemcitabine. N Engl J Med 2013; 369: 1691-703.

11. Portal A, Pernot S, Tougeron D, et al. Nab-paclitaxel plus gemcitabine for metastatic pancreatic adenocarcinoma after Folfirinox failure: an AGEO prospective multicentre cohort. Br J Cancer 2015; 113: 989-95.

12. Wang-Gillam A, Li C-P, Bodoky G, et al. Nanoliposomal irinotecan with fluorouracil and folinic acid in metastatic pancreatic cancer after previous gemcitabine-based therapy (NAPOLI-1): a global, randomised, open-label, phase 3 trial. The Lancet 2016; 387: 54557.

13. Akhoundova Sanoyan D, Reiner CS, Papageorgiou P, Siebenhüner AR. Sequential treatment of metastatic adenocarcinoma of the pancreatic duct with liver metastasis following the NAPOLI-1 study protocol with nal-irinotecan plus 5-FU in the second line. Case Reps Oncol 2020; 13: 79-84.

14. Nielson CM, Bylsma LC, Fryzek JP, Saad HA, Crawford J. Relative dose intensity of chemotherapy and survival in patients with advanced stage solid tumor cancer: a systematic review and metaanalysis. Oncologist. 2021;9:1609-18.

15. McMillan DC, Watson WS, O'Gorman P, Preston T, Scott HR, McArdle CS. Albumin concentrations are primarily determined by the body cell mass and the systemic inflammatory response in cancer patients with weight loss. Nutr Cancer. 2001; 2: 210-3.

16. Meyer EJ, Nenke MA, Rankin W, Lewis JG, Torpy DJ. Corticosteroid-binding globulin: a review of basic and clinical advances. Horm Metab Res 2016; 6: 359-71.

17. Lv GY, An L, Sun XD, Hu YL, Sun DW. Pretreatment albumin to globulin ratio can serve as a prognostic marker in human cancers: a meta-analysis. Clin Chim Acta 2018; 476: 81-91.
18. Cherri S, Noventa S, Zaniboni A. Pancreatic adenocarcinoma: Beyond first line, where are we? World J Gastroenterol 2021; 3: 1847-63.

19.Leader A, Hofstetter L, Spectre G. Challenges and advances in managing thrombocytopenic cancer patients. J Clin Med. 2021; 10: 1169.

20. Turker S, Cilbir E, Guven DC, Karacin C, Yalcin S. The relation between inflammation-based parameters and survival in metastatic pancreatic cancer. J Cancer Res Ther. 2021; 17: 510-5.

21.Toledano-Fonseca M, Cano MT, Inga E, et al. The combination of neutrophil-lymphocyte ratio and platelet-lymphocyte ratio with liquid biopsy biomarkers improves prognosis prediction in metastatic pancreatic cancer. Cancers (Basel) 2021; 13: 1210.

22.Tezuka K, Okamura Y, Sugiura T, et al. Predictive factors of survival in patients with borderline resectable pancreatic cancer who received neoadjuvant therapy. Pancreatology. 2021;8:1424903.

23. Pradere B, D'Andrea D, Schuettfort VM, et al. UTUC collaboration. Pre-therapy serum albumin-to-globulin ratio in patients treated with neoadjuvant chemotherapy and radical nephroureterectomy for upper tract urothelial carcinoma. World J Urol. 2021;7:256777.

24. Feng L, Gu S, Wang P, et al. Pretreatment values of bilirubin and albumin are not prognostic predictors in patients with advanced pancreatic cancer. Cancer Med 2018; 7: 5943-51.

25.Liu J, Chen S, Geng Q, et al. Prognostic value of pretreatment albumin-globulin ratio in predicting long-term mortality in gastric cancer patients who underwent D2 resection Onco Targets Ther 2017; 10: 2155-62. 